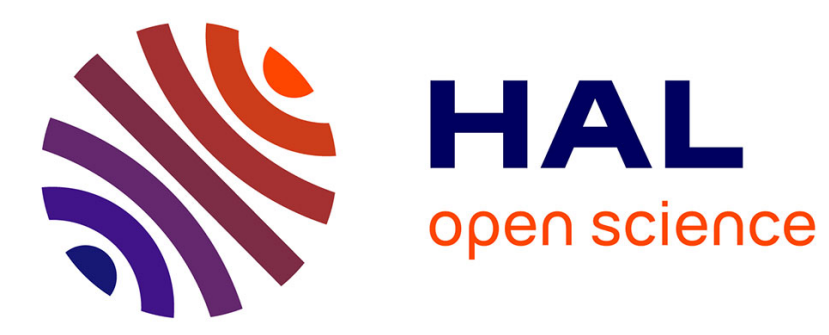

\title{
La faune de la galerie rive droite du Mas d'Azil (Ariège) : données paléoclimatiques et paléthnographiques
}

\author{
Marylène Patou-Mathis
}

\section{To cite this version:}

Marylène Patou-Mathis. La faune de la galerie rive droite du Mas d'Azil (Ariège) : données paléoclimatiques et paléthnographiques. Bulletin de la Société préhistorique française, 1984, 81 (10-12), pp.311-319. halshs-00387042

\section{HAL Id: halshs-00387042 \\ https://shs.hal.science/halshs-00387042}

Submitted on 22 May 2009

HAL is a multi-disciplinary open access archive for the deposit and dissemination of scientific research documents, whether they are published or not. The documents may come from teaching and research institutions in France or abroad, or from public or private research centers.
L'archive ouverte pluridisciplinaire $\mathbf{H A L}$, est destinée au dépôt et à la diffusion de documents scientifiques de niveau recherche, publiés ou non, émanant des établissements d'enseignement et de recherche français ou étrangers, des laboratoires publics ou privés. 


\title{
La faune de la Galerie Rive Droite du Mas d'Azil (Ariège) : données paléoclimatiques et paléthnographiques
}

\author{
par Marylène Patou
}

Les fouilles effectuées au Mas d'Azil dans la Galerie Rive Droite (de l'Arize) par Marthe et Saint-Just Péquart de 1937 à 1943 ont livré un matériel de l'époque magdalénienne riche en art mobilier, en industries lithique et osseuse et en faune. Les inventeurs ont donné une étude, qui n'était pas exhaustive, du matériel (Péquart M. et Péquart S.-J., 1960, 1961, 1962). Elle a été complétée en ce qui concerne l'industrie lithique et les œuvres d'art par Des Hermens de Bayle et Vialou (1979). La faune restait à publier. Ces pages font suite à la communication en séance du 9 octobre 1982 (1).

\section{ÉTUDE PALÉOZOOLOGIQUE}

\section{Espèces déterminées}

Nous n'exposerons pas ici la démarche paléontologique suivie pour aboutir à la détermination de toutes les espèces présentes dans ce matériel, nous donnons directement les résultats.

Il consiste en 2159 restes déterminés, essentiellement de grands mammifères et d'oiseaux qui ont été étudiés par Philippe Vilette.

- Il s'agit de pièces triées par les fouilleurs ; les esquilles sont rares. La matériel est regroupé en un seul ensemble attribuable, d'après les données des auteurs, au Magdalénien.

(1) B.S.P.F., 79, p. 195.
La liste de faune est la suivante :

\begin{tabular}{lrr}
\multicolumn{1}{c}{ ESPÈCES } & NR & NMI \\
Rangifer tarandus & 1268 & 92 \\
Cervus elaphus & 464 & 24 \\
Equus caballus & 174 & 12 \\
Bos primigenius & 17 & 7 \\
Bison priscus & 9 & 2 \\
Bos ou Bison & 150 & 15 \\
Capreolus capreolus & 2 & 1 \\
Rupicapra rupicapra & 13 & 3 \\
Capra ibex pyrenaïca & 20 & 4 \\
Sus scrofa & 2 & 1 \\
Canis lupus & 11 & 1 \\
Ursus spaeleus & 4 & 1 \\
Crocuta spelaea & 4 & 2 \\
Vulpes vulpes & 20 & 5 \\
Oryctolagus cuniculus & 1 & 1
\end{tabular}

\section{L'âge des individus}

Deux espèces nous ont permis de déterminer avec une certaine précision (le nombre de restes étant suffisant) l'âge des individus observés dans le matériel, le Renne et dans une moindre mesure le Cerf.

\section{Le Renne}

Nous nous sommes fondée sur les travaux de J. Bouchud (1966). L'étude de la dentition déciduale a permis de constater la présence d'au moins 31 jeunes (de 1 mois à 3 ans) dont l'âge est donné dans 
le tableau I. Celle de la dentition définitive nous conduit à reconnaître 61 adultes qui se répartissent dans les classes d'âge définies tableau II.

Le Renne est donc représenté au Mas d'Azil par au moins 92 individus.

\section{Le Cerf élaphe}

Nous avons suivi la même démarche pour identifier les restes dentaires des cerfs.

L’âge des sujets a été déterminé, sur les mandibules et les dents isolées inférieures, à partir des données de Riglet (1977).

Pour les jeunes cerfs (de la naissance à deux ans), les résultats sont exprimés dans le tableau III.

Pour les adultes (âgés de plus de deux ans) la répartition est donnée dans le tableau IV.

Le Cerf élaphe est donc représenté au Mas d'Azil par au moins 24 individus.

\section{Pourcentage relatif des espèces}

Le tableau $\mathrm{V}$ résume toutes les données concernant le dénombrement de la grande faune.

Les diagrammes circulaires, aussi bien pour les N.M.I. (Figure 1) que pour les N.R. (Figure 2), montrent une nette dominante du Renne suivi du Cerf et des Bovinés, pour un N.M.I. total estimé à 171 et un N.R. de 2159.

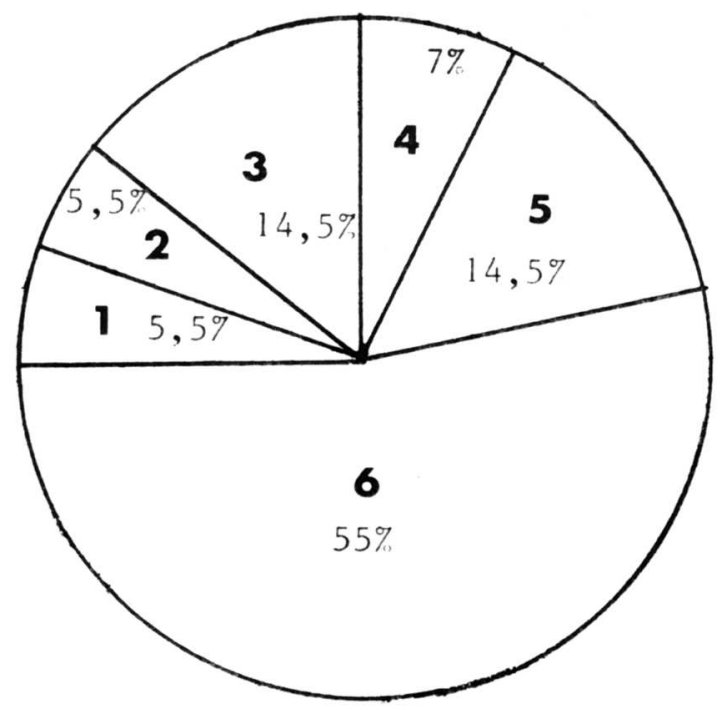

Fig. 1 - Pourcentage relatif des espèces au Mas d'Azil (G.R.D.), en fonction du N.M.I.

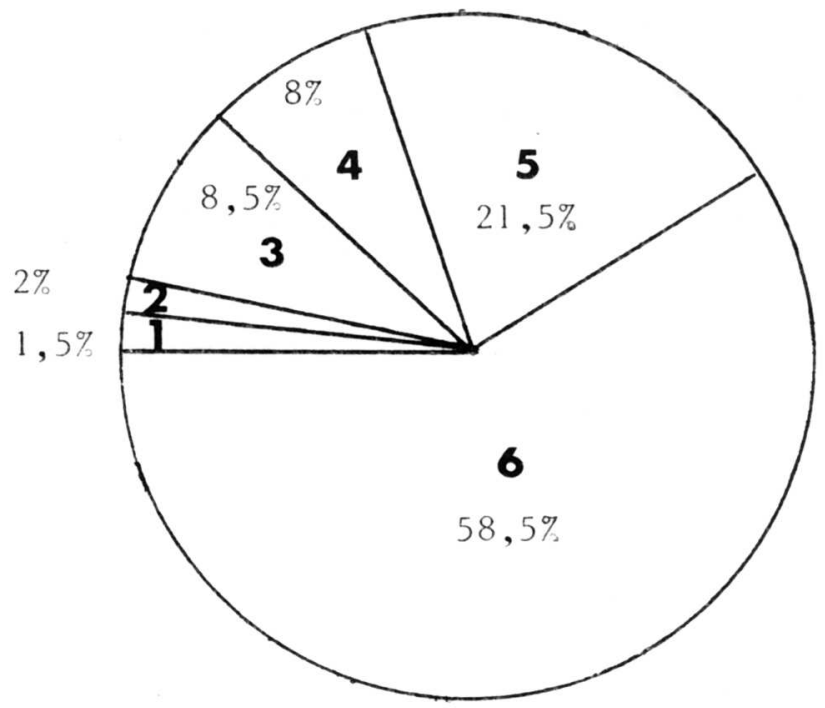

Fig. 2 - Pourcentage relatif des espèces au Mas d'Azil (G.R.D.), en fonction du N.R.

\section{ÉTUDE PALETHNOGRAPHIQUE}

\section{Animaux chassés et consommés}

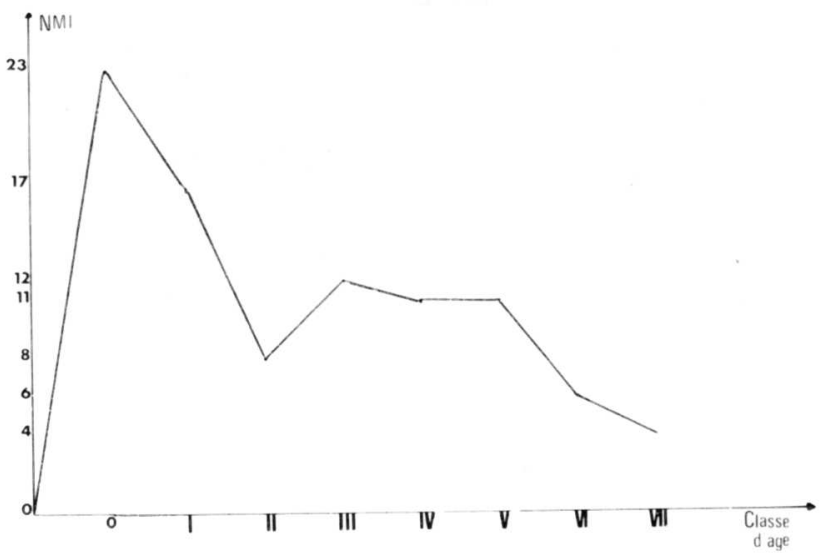

Fig. 3 - Courbe de mortalité des Rennes du Mas d'Azil (G.R.D.).

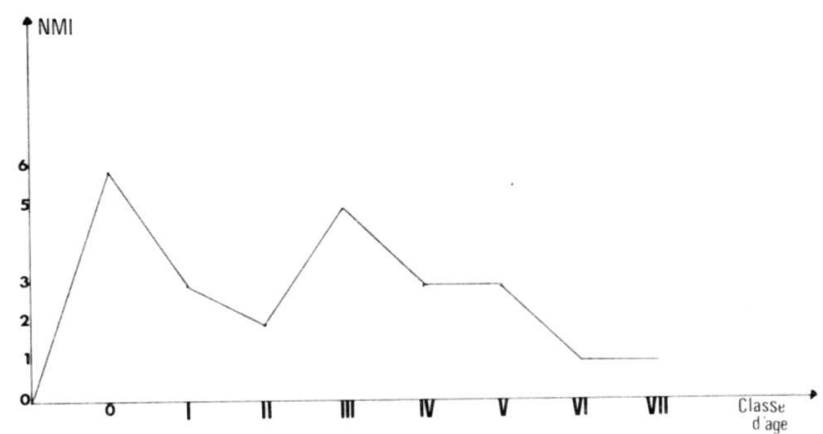

Fig. 4 - Courbe de mortalité des Cerfs du Mas d'Azil (G.R.D.). 
Nous avons pu tracer grâce aux calculs pour chaque classe d'âge du N.M.I., les courbes de mortalité du Renne (Figure 3) et du Cerf (Figure 4), celle-ci est à la limite de la validité statistique le N.R., étant de 24. Elles correspondent à des courbes de chasse (nombreux animaux adultes abattus).

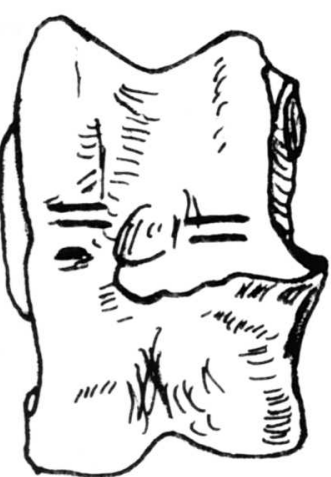

b

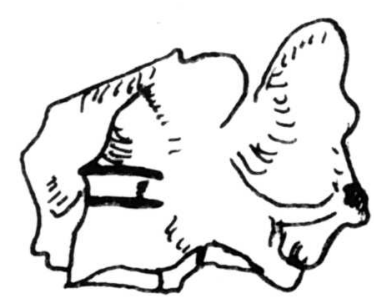

a 1

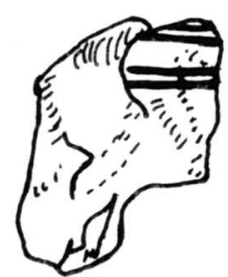

d

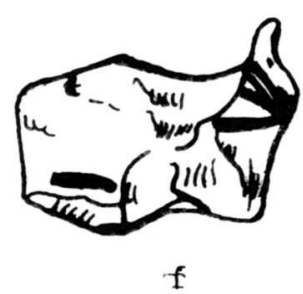

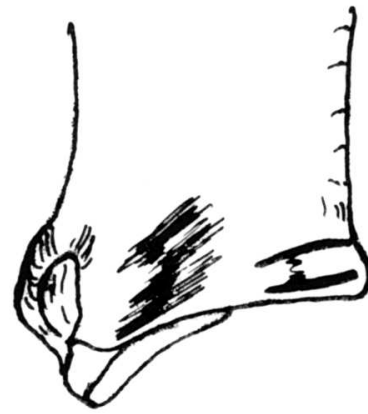

a

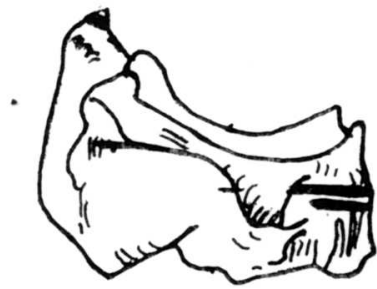

$c^{2}$

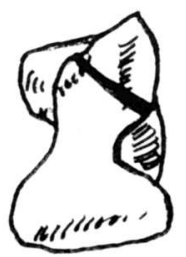

e

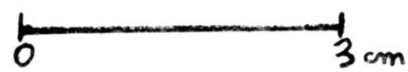

Fig. 5 - Localisation des stries sur les os des Rennes du Mas d'Azil (G.R.D.). a : scapula droite, vue latérale, b : talus droit, vue antérieure, c : cubo-naviculaire droit, 1 : vue antérieure, 2 : vue latérale externe, d : pyramidal droit, vue latérale externe, e: semi-lunaire droit, vue supérieure, $f$ : scaphoide, vue latérale interne.

\section{Brûlures}

Dans un matériel Paléolithique les os calcinés, contrairement à certaines faunes mésolithiques, sont peu nombreux.

Dans ce site on a $0,5 \%$ d'ossements brûlés. Ils appartiennent tous aux éléments du squelette postcrânien, ce sont surtout les os du carpe, du tarse et les phalanges (tableau VII).

Les brûlures se situent sur les parties distales des membres, les morceaux de viande (épaule et cuissot) laissaient en contact direct avec le feu des extrémités pauvres en viande, d'où les marques observées.

Marques de boucherie

L'étude des stries intentionnelles observables sur les os et notamment leur localisation, apporte des données importantes pour la compréhension des diverses étapes du traitement d'un gibier par les préhistoriques. Le tableau VI résume les observations faites sur le matériel de ce gisement.

Nous constatons la présence des témoins de trois principales opérations :

- « dépouillage », surtout aux niveaux des maxillaires.

- désarticulation et "décarnisation ", sur les os longs des membres. Le pourcentage des os comportant des marques est de $3(45 \%$ sur le squelette crânien et $55 \%$ sur le post-crânien) (Figure 5).

Les os ont-ils pu servir de combustible ? Il est impossible dans le matériel du Mas d'Azil de le savoir puisqu'il n'y a aucune preuve directe, aucun ossement ne présente une calcination poussée comme celle que l'on peut observer expérimentalement. Si tel fut le cas une carbonisation totale des os entraîne leur complète disparition ou transformation (cendres).

Conservation des éléments anatomiques des grands herbivores

Chez les quatre groupes: Renne, Cerf, Cheval, Bovinés, les dents sont les éléments les mieux conservés. Les métacarpes résistent mieux que les métatarses chez les Cervidés mais moins bien chez les Équidés et les Bovinés. Les os longs qui se conservent le mieux sont le radius et le tibia (Figure $6)$.

La conservation des os dépend de la taille, de la résistance mécanique aux pressions externes et de la soudure précoce ou non des épiphyses (Bouchud, 1966).

Le dénombrement des restes fauniques montre que la fréquence relative d'un os est d'autant plus grande que la soudure des épiphyses est plus précoce. 


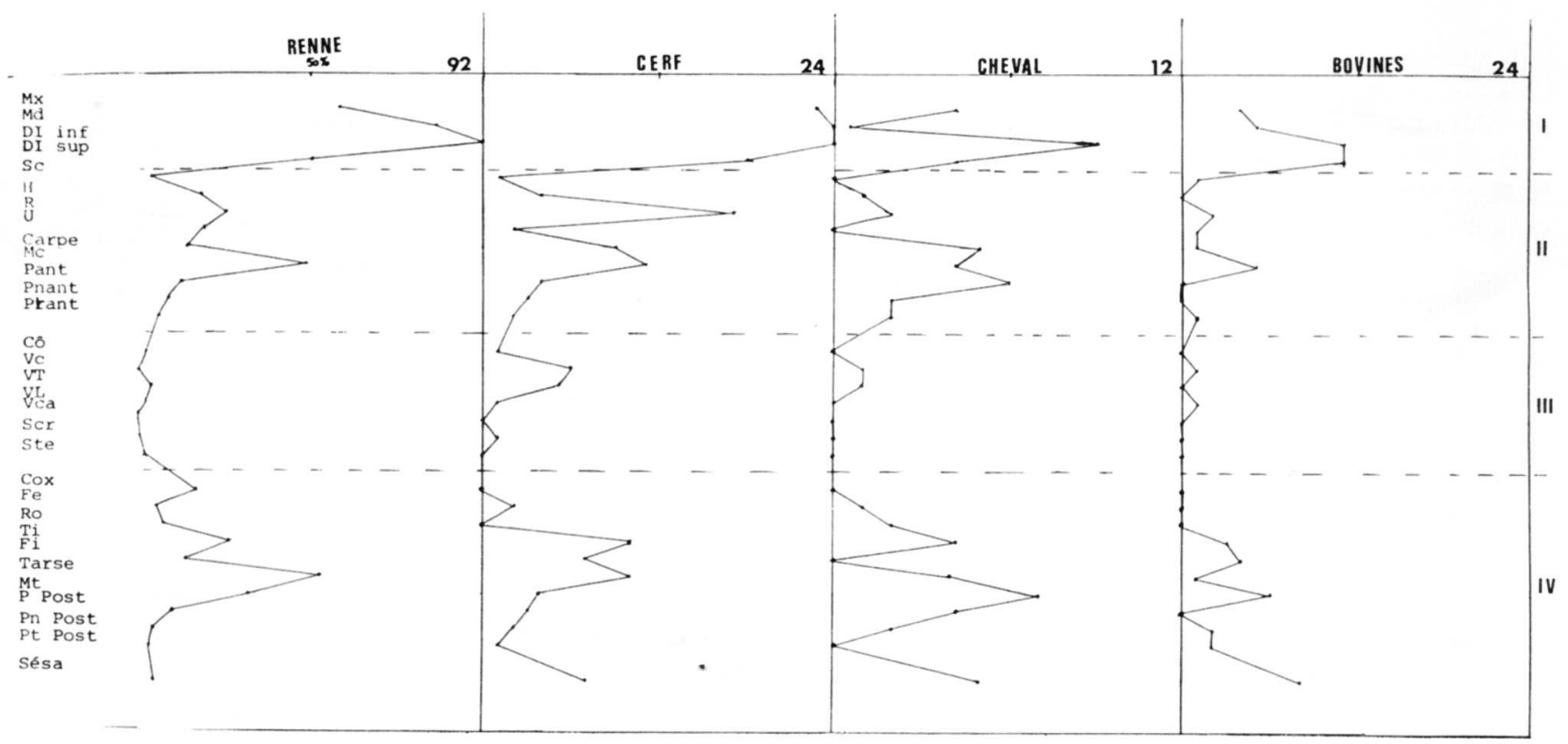

Fig. 6 - Conservation des éléments anatomiques des Rennes du Mas d'Azil (G.R.D.).

On constate dans ce matériel que les phalanges des Bovinés sont mal conservées, il est possible que ces dernières aient été fracturées pour en extraire la moëlle très riche en graisse pouvant servir d'huile d'éclairage.

Estimation du poids de viande fourni par les herbivores

L'estimation de l'apport carné de chaque espèce d'herbivore (en fonction du N.M.I.), permet d'avoir une idée de la consommation globale de viande. Elle est sûrement sous-estimée.

Le poids moyen de chaque espèce correspond à. des données de plusieurs auteurs. Ici la viande est fournie essentiellement par les Bovinés, les Cervidés et les Chevaux. Les autres espèces ne représentent qu'environ $1 \%$ du total (tableau VIII).

Occupation du site

La détermination de l'âge des jeunes rennes nous permet de tracer l'histogramme mensuel (Figure 7) pour cette espèce et donc de cerner les périodes d'occupation de la grotte.

Le Cerf nous indique la présence des chasseurs en septembre, octobre, décembre, mars ou avril et juin.

On constate d'après ces résultats que la grotte du Mas d'Azil fut une habitation quasi-permanente, on note une période intensive de chasse donc vraisemblablement d'occupation en automne-hiver (2).

(2) Il nous faut garder en mémoire l'épaisseır $(60 \mathrm{~cm})$ de la couche archéologique fouillée. Elle peut correspondre à plusieurs niveaux d'habitats.

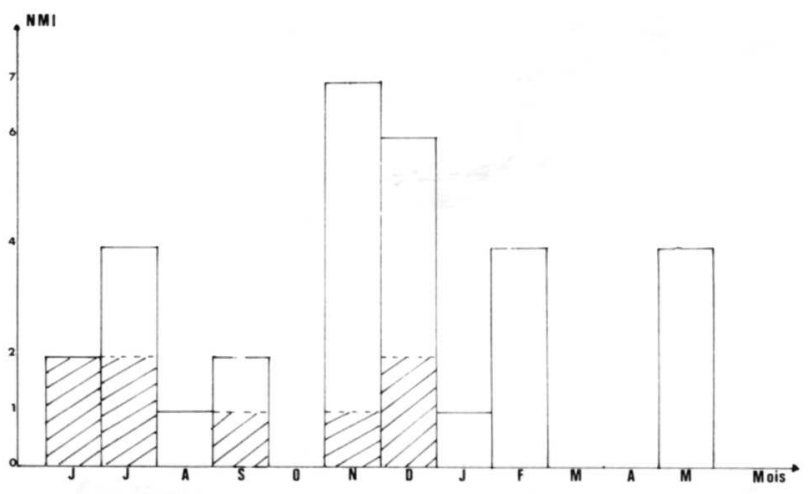

Fig. 7 - Histogramme mensuel, tracé à partir du N.-M. de jeunes rennes du Mas d'Azil (G.R.D.). Les hachurés correspondent aux animaux âgés de 2 à 3 ans, les résultats qu'ils fournissent sont plus incertains.

LES DIFFÉRENTES UTILISATIONS DE LA GRANDE FAUNE PAR L'HOMME PRÉHISTORIQUE DU MAS D'AZIL

\section{L'alimentation}

Le Renne, le Cerf et les Bovinés, furent les gibiers préférés des hommes du Mas d'Azil.

Les jeunes rennes représentent $50 \%$ des individus de cette espèce, ceci est fréquent dans les gisements Magdaléniens, dans les hardes ils ne représentent qu'environ $15 \%$.

La courbe de conservation différentielle des éléments anatomiques montre une abondance des restes dentaires et des extrémités des membres. Les os appartenant au squelette axial (côtes et vertèbres 
notamment), sont rares, on peut penser que le débitage de l'animal se faisait à l'extérieur de la galerie. Les restes du crâne sont absents (exceptés quelques fragments de bois), la boîte cranienne a dû subir une fracturation importante pour permettre d'en extraire la cervelle.

\section{Étude de la fragmentation : tableau IX}

On constate que tous les os longs ont été fracturés pour permettre d'en extraire la moëlle. Les omoplates et les os coxaux sont réduits à leurs seules portions articulaires. Les os les moins fragmentés sont ceux du tarse, du carpe et les phalanges (excepté chez le Cerf). La conservation et la fragmentation des os sont pratiquement identiques pour les quatre espèces.

La présence d'os calcinés, notamment de Renne, montre que les Magdaléniens faisaient rôtir certains morceaux de viande comme les «épaules » et les « cuissots ».

Le peu d'esquilles (10\% par rapport aux os déterminés, dont $80 \%$ appartiennent au crâne) ne permet malheureusement pas la reconstitution des os longs entiers, de ce fait nous ne pouvons pas retrouver avec certitude la technique de fracturation.

\section{Fabrication des vêtements}

On peut raisonnablement penser que les Préhistoriques utilisaient la peau des Cervidés, des Bovidés et la fourrure des Carnivores comme protection contre le froid.

Le matériel faunique ne permet aucune certitude à ce sujet, mais la découverte d'un outillage lithique et osseux (aiguilles à chas, perçoirs...) ayant pu avoir comme fonction la «couture " étayent notre hypothèse.

\section{L'industrie osseuse}

Une très abondante collection d'outils en os a été trouvée dans ce site par M. et St. J. Péquart. La matière première utilisée est essentiellement le bois de Cervidés mais également et en pourcentage non négligeable les éclats d'os longs.

\section{"Euvre d'art»}

Dans ce matériel nous avons découvert et étudié 24 os coxaux de Renne présentant une fracturation identique.

La cavité cotyloïde (ou acetabulum) est conservée, plus ou moins isolée, avec ablation de l'ilion, de l'ischion et du pubis, ce qui donne une stabilité parfaite à l'objet (Figure 8 ).

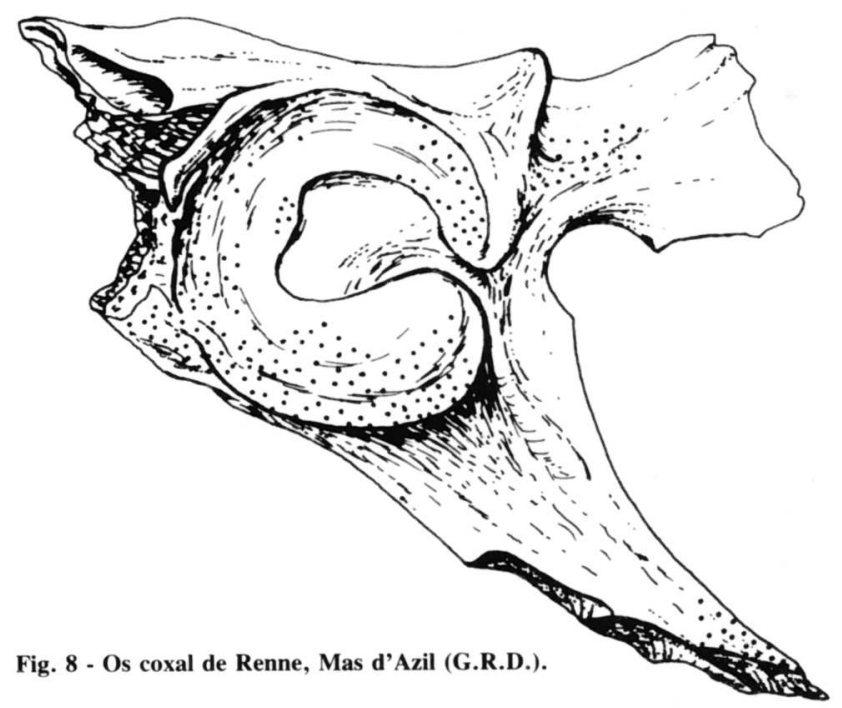

Il est intéressant de noter la présence dans 16 d'entre elles d'ocre rouge, s'agit-il de « godets à ocre »? (3).

Un de ces récipients présente une pointe retouchée (fragment d'ilion), aux angles émoussés et lustrés, avec à son extrémité un dépôt important d'ocre rouge. Nous pouvons émettre deux hypothèses :

- Cette pointe a pu servir à mélanger le liant et l'ocre.

- Elle a pu servir à la fabrication de poudre d'ocre à partir de nodules.

La découverte de plusieurs nodules ferrugineux taillés à pans et portant des traces laissées par l'outil (stries) qui a enlevé par raclage des parcelles de la matière sous forme de poudre, appuie la deuxième possibilité d'utilisation de cet objet.

De très nombreux éclats d'os ont été utilisés comme support à des gravures et des sculptures.

C'est une des raisons de l'absence de certains éléments anatomiques.

C'est le cas pour les rondelles gravées sur des omoplates de rennes et les os hyoides de chevaux servant de support aux contours découpés.

Il est intéressant de constater que les outils et les œuvres d'art (souvent fragmentés) se trouvaient mélangés aux déchets de cuisines (Marthe et St. Just Pequart, 1962).

\section{Les éléments de parure}

Les éléments de parure les plus importants par le nombre sont les dents percées, mais les coquilles, plus rares, témoignent également de certaines activités.

(3) M.-D. VIALOU m'informe de la présence en abondance d'ocre rouge sur les parois de cette galerie. 


\section{- Les dents percées}

Les incisives de chevaux, de rennes et de cerfs, ainsi que les canines de Carnivores, ont été employées pour la confection des parures des magdaléniens du Mas d'Azil.

Dans le matériel étudié, $25 \%$ des incisives lactéales de Renne présentent sur la face antérieure de la racine dans sa partie médiane une rayure profonde. F. Poplin (1983) avait étudié 12 incisives de Rennes provenant de ce matériel et qui comportaient également des incisions.

\section{Problèmes chronostratigraphique et paléoclimatolo- gique}

Différentes observations nous ont amenée à nous poser plusieurs questions concernant la stratigraphie de ce gisement.

- La présence en abondance du Cerf élaphe (21\%) avec le Renne (58\%) est, comparativement aux gisements du Sud-Ouest de la France de cette même période, une particularité. F. Delpech (1975) remarque que l'élaphe ne devait qu'exceptionnellement fréquenter la Haute Vallée de l'Ariège. Le gisement d'Enlène n'a donné, pour l'instant, aucun reste de Cerf, alors que le Renne est dominant.

- L'obtention d'un très mauvais coefficient de corrélation ( $\mathrm{N}=41, \mathrm{r}=0,302)$ entre la hauteur et la largeur mesurées sur les astragales de Renne, alors que ces deux variables sont habituellement fortement liées (Bouchud, 1966). Ceci peut correspondre à un mélange de population (migrations saisonnières, deux sous-espèces différentes...).

La reconstitution du paléoclimat et du paléoenvironnement à l'aide de la seule grande faune est une entreprise délicate. Le pourcentage relatif des espèces représente la chasse. C'est-à-dire une sélection du gibier par les magdaléniens pas la réalité de l'environnement. De plus on peut penser raisonnablement qu'ils se déplaçaient, pouvant ainsi rapporter du gibier d'une région plus éloignée de leur campement. Pour que la reconstitution soit la plus rigoureuse possible, elle doit s'accompagner d'autres études : pollens, sédiments... Les Rongeurs sont de très bons indicateurs mais ils n'ont pas été récoltés. Les restes d'oiseaux ont été étudiés par P. Vilette.

Le pourcentage d'Oiseaux d'espaces découverts et froid est très important $(65 \%)$, ce sont principalement des Lagopèdes et des Chocards. Les données précédentes et le tableau $\mathrm{X}$ nous amènent à nous interroger sur la chronostratigraphie du gisement.
Il est intéressant de noter que la végétation du Dryas ancien (15 000-12 $000 \mathrm{BP})$ correspond à un climat froid et sec dans un paysage découvert avec seulement un maximum de $2 \%$ d'arbres (Jalut, 1976).

L'oscillation de Bölling (1400-1200 BP) n'a pas été mise clairement en évidence sur le versant NordPyrénéen, elle se caractérise par une augmentation du pourcentage d'arbres (jusqu'à $30 \%$ ).

Les datations récentes de la Galerie données par Paul Bahn (communication orale) : $13400 \pm 100$ ans BP (GIF 5679) et $13200 \pm 110$ BP (GIF 5680).

La faune de la Galerie Rive Droite du Mas d'Azil pourrait être un des témoins de l'existence dans cette région de l'interstade de Bölling. Après les niveaux du Dryas ancien froid et sec avec peu d'arbres, dominance du Renne et peu ou pas de Cerfs, il y aurait eu un réchauffement du climat entraînant une augmentation du pourcentage d'arbres ainsi que :

- une apparition ou une augmentation du nombre de Cerfs (de plus petite taille?),

- l'apparition du Chevreuil, du Sanglier, et du Bœuf primitif,

- un changement de populations de Rennes (de plus petite taille ?).

Ces données restent des hypothèses, il serait intéressant de revoir les collections d'industries lithiques et osseuses de cette galerie et si possible de faire des études palynologique et sédimentologique (4).

L.A. 184, I.P.H., 1, rue R.-Panhard, 75013 Paris.

(4) Pour être sûr que nous n'avons pas superposition du Magdalénien IV et du Magdalénien VI, ce qui est très fréquent dans les gisements de cette région.

Bouchud J. (1953) - La mandibule de Renne, Mammalia, t $18, \mathrm{n}^{\circ} 1$, pp. $27-49,2$ pl., 4 fig., 4 tabl.

Bouchud J. (1954) - Le Renne et le problème des migrations. L'Anthropologie, t. 58, n' 1-2, pp. 79-85.

Bouchud J. (1966) - Essai sur le Renne et la climatologie du Paléolithique moyen et supérieur. Imprimerie Magne, Périgueux, 300 p., XIII pl, 55 fig., LXXI tabl.

Delpech F. (1975) - Les faunes du Paléolithique supérieur dans le Sud-Ouest de la France, Thèse de Doctorat d'État es Sciences Naturelles. Université de Bordeaux I, t. 1 : texte, 374 p., t. 2 : 159 tabl., t. $3: 98 \mathrm{pl}$.

Des Hermens R. de Bayle et Vialou D. (1979) - Étude d'une série magdalénienne inédite du Mas d'Azil (Ariège), L'Anthropologie, t. 83, $\mathrm{n}^{\circ} 4$, pp. 556-583.

JaLut G. (1976) - La végétation du Pléistocène Supérieur et au début de l'Holocène dans les Pyrénées, La Préhistoire Française, t. 1 , pp. $512-516$ 
Patou M. (1985) - Dénombrement et détermination de l'âge des Rennes (Rangifer tarandus) provenant de la galerie Rive Droite du Mas d’Azil (Ariège) : méthodes et résultats, Genève, Revue de Paléobiologie du Muséum d'Histoire Naturelle, vol. 4, $\mathrm{n}^{\circ} 1$.

Pequart M. et St. J. (1960) — La grotte du Mas d'Azil (Ariège) une nouvelle galerie magdalénienne, Annales de Paléontologie, t. 46, pp. 127-194.

Pequart M. et St. J. (1961) - Idem, t. 47, pp. 157-250.
Pequart M. et St. J. (1961) - Idem, t. 48, pp. 199-286.

Poplin F. (1983) - Incisives de Rennes sciées In La faune et l'Homme préhistoriques. Paris, Mémoire de la S.P.F., t. 16 , pp. 55-67, 9 fig.

Riglet P.-H. (1977) - Contribution à l'étude de l'âge du Cerf élaphe. Thèse pour le Doctorat de Vétérinaire, École Vétérinaire d'Alfort.

SPIESS A.-E. (1981) - Reindeer and Caribou hunters, an Archaeological Study, Academy de Press.

\begin{tabular}{|l|c|cccc|c|}
\hline \begin{tabular}{|l|l|l|l|}
\hline AGE EN \\
MOIS
\end{tabular} & 4 & 6 & 15 & $21-22$ & 24 & TOTAL. \\
\hline X.M.I. & 1 & 1 & 2 & 1 & 1 & 6 \\
\hline
\end{tabular}

Tableau III - Dénombrement, en fonction de l'âge, des jeunes cerfs du Mas d'Azil (G.R.D.)
Tableau I - Dénombrement, en fonction de l'âge, des jeunes rennes du Mas d'Azil (G.R.D.).
Tableau II - Dénombrement, par ciasse d'âge, des rennes adultes du Mas
d'Azil (G.R.D.).

Tableau II - Dénombrement, par ciasse d'âge, des rennes adultes du Mas
d'Azil (G.R.D.).

\begin{tabular}{|lccccccc|}
\begin{tabular}{lllllll|} 
CLASSES EN \\
ANEES
\end{tabular} & I(3-4) & $11(4-6)$ & III (6-8) & IV $(8-10)$ & V(10-12) & VI (12-13) & VII(>13) \\
\hline N.M.I. & 9 & 8 & 12 & 11 & 11 & 6 & 4 \\
\hline
\end{tabular}

Tableau IV - Dénombrement, par classe d'âge, des cerfs aduites du Mas
d'Azil (G.R.D.).

\begin{tabular}{|c|c|c|c|c|c|c|c|c|c|c|c|c|c|c|c|c|}
\hline \multicolumn{2}{|c|}{ ESPECES } & LAP IN & $\begin{array}{c}\text { BOUQUE } \\
\text {-TIN }\end{array}$ & ISARD & $\begin{array}{c}\text { CHEVRE } \\
\text {-UIL }\end{array}$ & $\begin{array}{l}\text { SAN- } \\
\text { GLIER }\end{array}$ & CHEVAL & BOEUF & BISON & $\begin{array}{l}\text { BOEUF } \\
\text { BISON }\end{array}$ & CERF & RENNE & OURS & HYENE & LOUP & RENARD \\
\hline \multicolumn{2}{|l|}{$\begin{array}{l}\alpha \\
z\end{array}$} & 1 & 20 & 13 & 2 & 2 & 174 & 17 & 9 & 150 & 464 & 1268 & 4 & 4 & 11 & 20 \\
\hline \multirow{2}{*}{ م. } & $\frac{\sigma}{\Sigma}^{x}$ & 0 & 0 & 7 & 0 & 0 & 4 & 5 & 11 & 5 & 9 & 10 & 0 & 50 & 0 & 55 \\
\hline & 点 & 0 & 45 & 16 & 0 & 0 & 52 & 36 & 11 & 71 & 40 & 38 & 50 & 50 & 36 & 20 \\
\hline \multicolumn{2}{|c|}{ 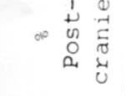 } & 100 & 55 & 77 & 100 & 100 & 44 & 59 & 78 & 24 & 51 & 52 & 50 & 0 & 64 & 25 \\
\hline \multirow{3}{*}{$\begin{array}{l}\longmapsto \\
\Sigma \\
z\end{array}$} & 7 & 0 & 0 & 1 & 0 & 0 & 5 & 0 & 0 & 6 & 6 & 31 & 0 & 0 & 0 & 0 \\
\hline & "ृ & 1 & 4 & 2 & 1 & 1 & 7 & 7 & 2 & 9 & 18 & 61 & 1 & 2 & 1 & 5 \\
\hline & H & 1 & 4 & 3 & 1 & 1 & 12 & 7 & 2 & 15 & 24 & 92 & 1 & 2 & 1 & 5 \\
\hline
\end{tabular}

Tableau V - Le nombre de restes (N.R.), le pourcentage relatif des éléments du squelette crânien et post-crânien (en fonction du N.R.) et le nombre minimal d'individus (N.M.I.) pour chaque espèce du Mas d'Azil (G.R.D.), D.I. : Dent Isolée. 


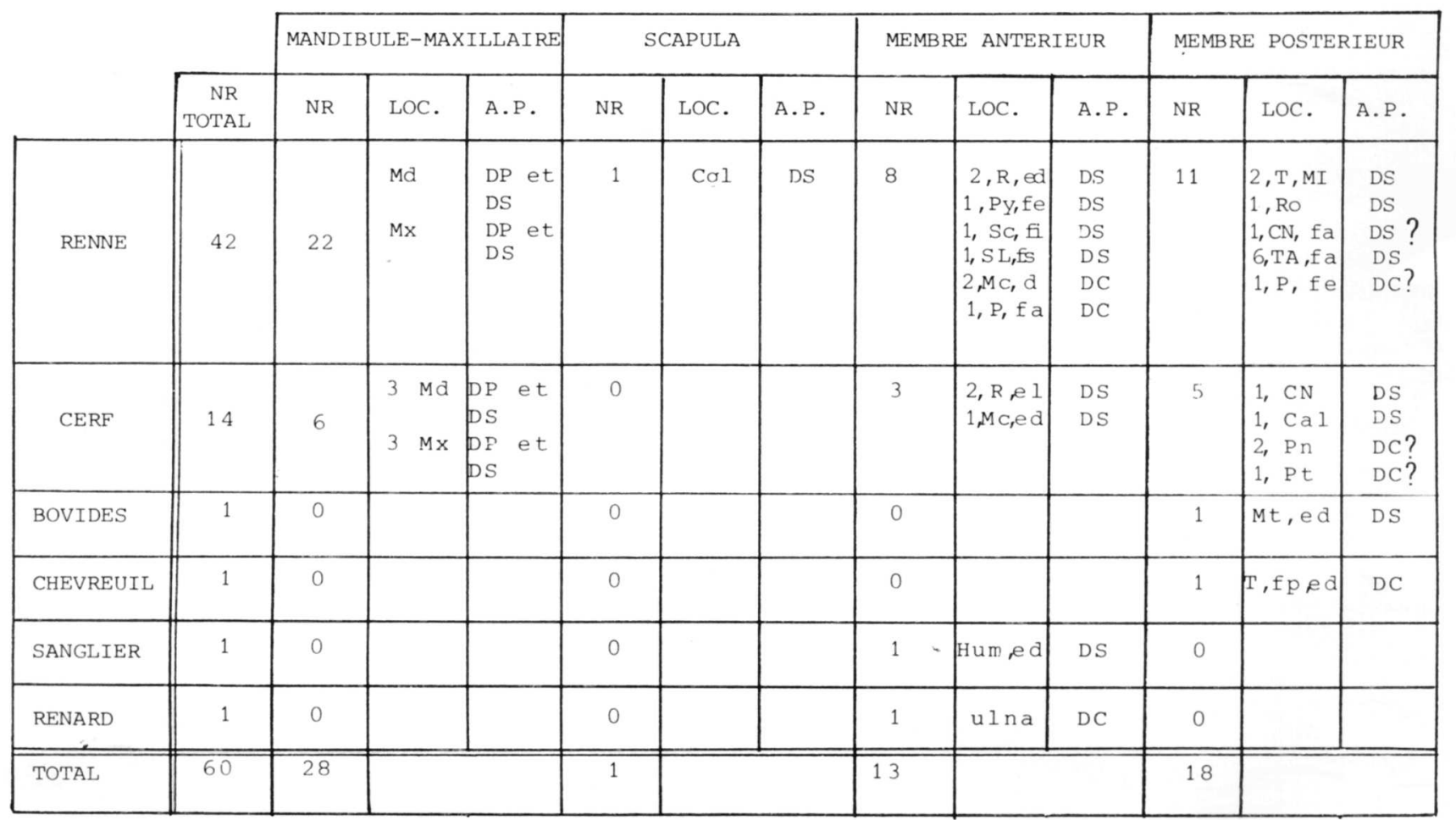

Tableau VI — Localisation des marques sur les ossements des mammifères du Mas d'Azil (G.R.D.) ; A.P. : Action Présumée ; Cal. : Calcaneum ; Cgl. : Cavité glénoìde ; CN. : Cubo-Naviculaire ; DC. : « Décarnisation »; DP. : « Dépouillage "; DS. : Désarticulation ; d. : diaphyse ; ed. : extrémité distale ; fa. : face antérieure ; fe. : face externe ; fi. : face interne ; fp. : face postérieure ; fs. : face supérieure ; Hum. : Humérus ; Mc. : Métacarpe ; Md. : Mandibule ; MI : Malléole Interne ; Mt. : Métatarse ; Mx. : Maxillaire ; P. : Phalange ; Pn. : Phalangine ; Pt. : Phalangette ; Py. : Pyramidal ; R. : Radius ; Ro. : Rotule ; Sc. : Scaphoïde ; SL. : Semi-Lunaire ; Ta. : Talus ; T. : Tibia.

\begin{tabular}{|c|c|c|c|}
\hline ESPECES & N.R. & LOCALISATION & $\begin{array}{l}\text { TOTALEMENT ov } \\
\text { PARTIELLEMENT }\end{array}$ \\
\hline RENNE & 7 & $\begin{array}{l}\text { Radius (ed) } \\
\text { Capitzato-trapézoïde } \\
\text { Unciforme } \\
\text { Phalange antérieure } \\
\text { Cubo-naviculaire } \\
\text { Talus } \\
\text { Phalange postérieure }\end{array}$ & $\begin{array}{c}\mathrm{T} \\
\mathrm{T} \\
\mathrm{T} \\
1 / 2 \\
\mathrm{~T} \\
\mathrm{~T} \\
\mathrm{~T}\end{array}$ \\
\hline CERF & 1 & Tibia (ed) & p \\
\hline ISARD & 1 & Phal ange postérieure & $\mathrm{T}$ \\
\hline TOTAL. & 9 & & \\
\hline
\end{tabular}

Tableau VII - Localisation des marques de calcination sur les ossements des mammifères du Mas d'Azil (G.R.D.).

\begin{tabular}{|c|c|c|}
\hline Milieu & Froid & Temperes \\
\hline Ouvert & $\begin{array}{l}\text { RENNE-CHEVAL-BISON } \\
65 \text { d'oiseaux }\end{array}$ & 6 'd'o1 seaux \\
\hline Forestier & CERF & $\begin{array}{c}\text { CERF-SANGLIER-BOEUF } \\
2 \text { d'oiseaux }\end{array}$ \\
\hline Rupicole & $\begin{array}{l}\text { BOUQUETIN-ISARD } \\
7 \text { d'oi seaux }\end{array}$ & \\
\hline
\end{tabular}

\begin{tabular}{|l|c|c|c|c|c|c|}
\hline ESPECES & NMI & NMI & \multicolumn{2}{|c|}{ POIDS DE VIANDE } & POIDS TOTAL & J \\
\hline JEUNES & ADULTES & \multicolumn{2}{|c|}{ JEUNES } & ADULTES & ESTIME & RELATIF \\
\hline RERF & 31 & 61 & 35 & 80 & 5965 & 22.4 \\
\hline CHEVAL & 5 & 18 & 70 & 150 & 3120 & 11.8 \\
\hline BOUQUETIN & 0 & 4 & 22 & 55 & 220 & 0.8 \\
\hline BOVIDES & 6 & 18 & 275 & 770 & 15510 & 58.5 \\
\hline ISARD & 1 & 2 & & 19 & 57 & 0.2 \\
\hline SANGLIER & 0 & 1 & & 60 & 60 & 0.2 \\
\hline CHEVREUIL & 0 & 1 & & 13 & 13 & 0.05 \\
\hline LAPIN & 0 & 1 & & 2 & 2 & 0.01 \\
\hline TOTAL & & & & & 26713 & \\
\hline
\end{tabular}

Tableau VIII - Estimation du poids de viande (en $\mathrm{Kg}$ ) fourni par les mammifères du Mas d'Azil (G.R.D.).

Tableau X : Écologie de certaines espèces présentes au Mas d'Azil (G.R.D.). 


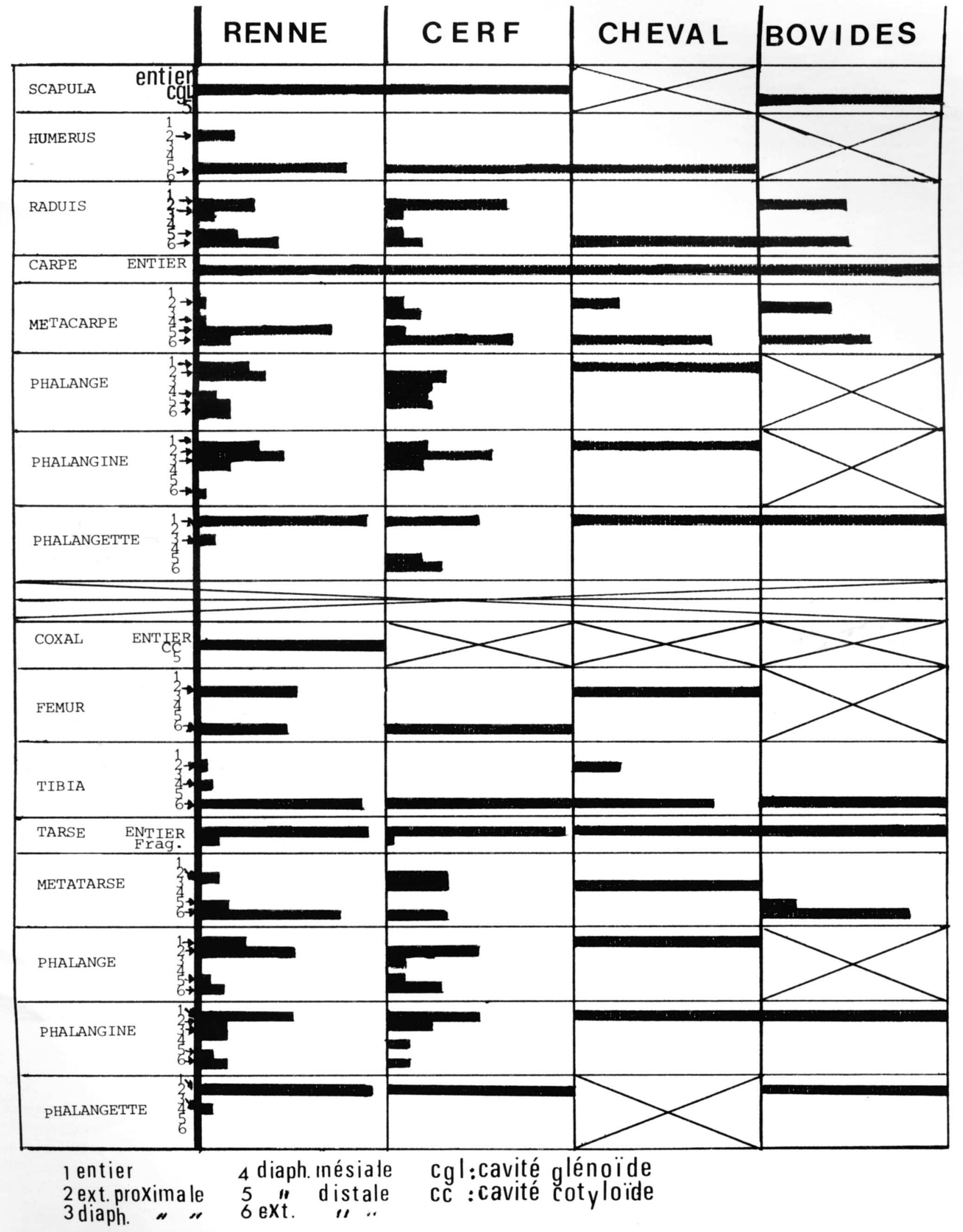

Tableau IX — Fragmentation des os chez le Renne, le Cerf, le Cheval et les Bovinés du Mas d'Azil (G.R.D.). 